\title{
ON SEMI GENERALIZED STAR $b$-CLOSED SET IN TOPOLOGICAL SPACES
}

\author{
S. Sekar ${ }^{1 \S}$, B. Jothilakshmi ${ }^{2}$ \\ ${ }^{1}$ Department of Mathematics \\ Government Arts College (Autonomous) \\ Salem, 636 007, Tamil Nadu, INDIA \\ ${ }^{2}$ Department of Mathematics \\ Government Arts College (Autonomous) \\ Coimbatore, 641 045, Tamil Nadu, INDIA
}

\begin{abstract}
In this paper, we introduce a new class of sets called semi generalized star $b$-closed sets in topological spaces (briefly $s g^{*} b$-closed set). Also we discuss some of their properties and investigate the relations between the associated topology.
\end{abstract}

AMS Subject Classification: 54A05

Key Words: $s g^{*} b$-closed set, $b$-closed set and $g b$ closed set

\section{Introduction}

In 1970, Levine introduced the concept of generalized closed set and discussed the properties of sets, closed and open maps, compactness, normal and separation axioms. Later in 1996 Andrjivic gave a new type of generalized closed set in topological space called $b$ closed sets. The investigation on generalization of closed set has lead to significant contribution to the theory of separation axiom, generalization of continuity and covering properties. A.A. Omari and M.S.M. Noorani made an analytical study and gave the concepts of generalized b closed sets in topological spaces.

Received: $\quad$ September 3, 2016

Revised: $\quad$ October 19, 2016

Published: December 17, 2016

$\S_{\text {Correspondence author }}$ (c) 2016 Academic Publications, Ltd. url: www.acadpubl.eu 
In this paper, a new class of closed set called semi generalized star $b$-closed set is introduced to prove that the class forms a topology. The notion of semi generalized star $b$-closed set and its different characterizations are given in this paper. Throughout this paper $(X, \tau)$ and $(Y, \sigma)$ represent the non-empty topological spaces on which no separation axioms are assumed, unless otherwise mentioned.

Let $A \subseteq X$, the closure of $A$ and interior of $A$ will be denoted by $\operatorname{cl}(A)$ and $\operatorname{int}(A)$ respectively, union of all $b$-open sets $X$ contained in $A$ is called $b$-interior of $A$ and it is denoted by $\operatorname{bint}(A)$, the intersection of all $b$-closed sets of $X$ containing $A$ is called $b$-closure of $A$ and it is denoted by $b c l(A)$.

\section{Preliminaries}

Definition 2.1. Let A subset $A$ of a topological space $(X, \tau)$, is called

1) a pre-open set [16] if $A \subseteq \operatorname{int}(\operatorname{cl}(A))$.

2) a semi-open set [13] if $A \subseteq \operatorname{cl}(\operatorname{int}(A))$.

3) a $\alpha$-open set [17] if $A \subseteq \operatorname{int}(\operatorname{cl}(\operatorname{int}(A)))$.

4) a $\alpha$ generalized closed set (briefly $\alpha g$ - closed) [14] if $\alpha c l(A) \subseteq U$ whenever $A \subseteq U$ and $U$ is open in $X$.

5) a generalized $*$ closed set (briefly $g^{*}$-closed) [20] if $\operatorname{cl}(A) \subseteq U$ whenever $A \subseteq U$ and $U$ is $\hat{g}$ open in $X$.

6) a generalized $b$ - closed set (briefly $g b$ - closed) [2] if $b c l(A) \subseteq U$ whenever $A \subseteq U$ and $U$ is open in $X$.

7) a generalized semi-pre closed set (briefly $g s p$-closed) [9] if $\operatorname{spcl}(A) \subseteq U$ whenever $A \subseteq U$ and $U$ is open in $X$.

8) a generalized pre- closed set (briefly $g p$ - closed) [10] if $p c l(A) \subseteq U$ whenever $A \subseteq U$ and $U$ is open in $X$.

9) a generalized semi- closed set (briefly $g s$ - closed) [9] if $\operatorname{scl}(A) \subseteq U$ whenever $A \subseteq U$ and $U$ is open in $X$.

10) a semi generalized closed set (briefly $s g$ - closed) [6] if $\operatorname{scl}(A) \subseteq U$ whenever $A \subseteq U$ and $U$ is semi open in $X$. 
11) a generalized pre regular closed set (briefly $g p r$-closed) [10] if $\operatorname{pcl}(A) \subseteq U$ whenever $A \subseteq U$ and $U$ is regular open in $X$.

12) a semi generalized $b$ - closed set (briefly $s g b$ - closed) [11] if $b c l(A) \subseteq U$ whenever $A \subseteq U$ and $U$ is semi open in $X$.

13) a $\ddot{g}$-closed set [19] if $\operatorname{cl}(A) \subseteq U$ whenever $A \subseteq U$ and $U$ is $s g$ open in $X$.

\section{Semi Generalized $b$ Star-Closed Sets}

In this section, we introduce semi generalized star $b$ - closed set and investigate some of its properties.

Definition 3.1. A subset $A$ of a topological space $(X, \tau)$, is called semi generalized star $b$ - closed set (briefly $s g^{*} b$-closed set) if $b c l(A) \subset U$ whenever $A \subset U$ and $U$ is $s g$ open in $X$.

Theorem 3.2. Every closed set is $s g^{*} b$-closed.

Proof. Let $A$ be any closed set in $X$ such that $A \subset U$, where $U$ is $s g$ open. Since $b c l(A) \subset \operatorname{cl}(A)=A$. Therefore $b c l(A) \subset U$. Hence $A$ is $s g^{*} b$-closed set in $X$.

The converse of above theorem need not be true as seen from the following example.

Example 3.3. Let $X=\{a, b, c\}$ with $\tau=\{X, \phi,\{b\},\{a, b\}\}$. The set $\{a, b\}$ is $s g^{*} b$ - closed set but not a closed set.

Theorem 3.4. Every $\ddot{g}$ - closed set is $s g^{*} b$-closed set.

Proof. Let $A$ be any $\ddot{g}$ - closed set in $X$ and $U$ be any $s g$ open set containing $A$. Then $b c l(A) \subset \operatorname{cl}(A) \subset U$. Therefore $b c l(A) \subset U$. Hence $A$ is $s g^{*} b$-closed set.

The converse of above theorem need not be true as seen from the following example.

Example 3.5. Let $X=\{a, b, c\}$ with $\tau=\{X, \phi,\{a, b\}\}$. The set $\{a, c\}$ is $s g^{*} b$ - closed set but not a $\ddot{g}$ - closed set.

Theorem 3.6. Every semi closed set is $s g^{*} b$-closed set. 
Proof. Let $A$ be any semi closed set in $X$ and $U$ be any $s g$ open set containing $A$. Since $A$ is semi closed set, $b c l(A) \subset \operatorname{scl}(A) \subset U$. Therefore $b c l(A) \subset U$. Hence $A$ is $s g^{*} b$ closed set.

The converse of above theorem need not be true as seen from the following example.

Example 3.7. Let $X=\{a, b, c\}$ with $\tau=\{X, \phi,\{a, b\}\}$. The set $\{b, c\}$ is $s g^{*} b$ - closed set but not a semi closed set.

Theorem 3.8. Every $\alpha$ - closed set is $s g^{*} b$-closed set.

Proof. Let $A$ be any $\alpha$ - closed set in $X$ and $U$ be any $s g$ open set containing $A$. Since $A$ is $\alpha-\operatorname{closed}, \operatorname{bcl}(A) \subset \alpha c l(A) \subset U$. Therefore $b c l(A) \subset U$. Hence $A$ is $s g^{*} b$-closed set.

The converse of above theorem need not be true as seen from the following example.

Example 3.9. Let $X=\{a, b, c\}$ with $\tau=\{X, \phi,\{b\},\{a, b\}\}$. The set $\{a, b\}$ is $s g^{*} b$ - closed set but not a $\alpha$-closed set.

Theorem 3.10. Every pre-closed set is $s g^{*} b$-closed set.

Proof. Let $A$ be any pre -closed set in $X$ and $U$ be any $s g$ open set containing $A$. Since every $A$ pre close set, $b c l(A) \subset \operatorname{pcl}(A) \subset U$. Therefore $b c l(A) \subset U$. Hence $A$ is $s g^{*} b$-closed set.

The converse of above theorem need not be true as seen from the following example.

Example 3.11. Let $X=\{a, b, c\}$ with $\tau=\{X, \phi,\{a\},\{b\}$, $\{a, b\}\}$. The set $\{a\}$ is $s g^{*} b$-closed set but not a pre-closed set.

Theorem 3.12. Every $\alpha g$-closed set is $s g^{*} b$-closed set.

Proof. Let $A$ be $\alpha g$-closed set in $X$ and $U$ be any open set containing $A$. Since every open set is $\operatorname{sg}$-open sets, we have $\operatorname{bcl}(A) \subset \alpha c l(A) \subset U$. Therefore $\operatorname{bcl}(A) \subset U$. Hence $A$ is $s g^{*} b$ - closed set.

The converse of above theorem need not be true as seen from the following example.

Example 3.13. Let $X=\{a, b, c\}$ with $\tau=\{X, \phi,\{a, b\}\}$. The set $\{b\}$ is $s g^{*} b$ - closed set but not a $\alpha g$ - closed set.

Theorem 3.14. Every $s g^{*} b$ - closed set is $g s p$ - closed set. 
Proof. Let $A$ be any $s g^{*} b$-closed set such that $U$ be any open set containing $A$. Since every open set is $\operatorname{sg}$-open, we have $b c l(A) \subset \operatorname{spcl}(A) \subset U$. Therefore $b c l(A) \subset U$. Hence $A$ is $g s p$-closed set.

The converse of above theorem need not be true as seen from the following example.

Example 3.15. Let $X=\{a, b, c\}$ with $\tau=\{X, \phi,\{a\},\{a, c\}\}$. The set $\{a, b\}$ is $g s p$ - closed set but not a $s g^{*} b$ - closed set.

Theorem 3.16. Every $s g^{*} b$ - closed set is $g b$ - closed set.

Proof. Let $A$ be any $s g^{*} b$-closed set in $X$ such that $U$ be any open set containing $A$. Since every open set is $s g$ open, we have $\operatorname{bcl}(A)$. Hence $A$ is $g b$-closed set.

The converse of above theorem need not be true as seen from the following example.

Example 3.17. Let $X=\{a, b, c\}$ with $\tau=\{X, \phi,\{a\},\{a, b\}\}$. The set $\{a, c\}$ is $g b$-closed set but not a $s g^{*} b$ - closed set.

Theorem 3.18. Every $s g$ - closed set is $s g^{*} b$ - closed set.

Proof. Let $A$ be any $s g$-closed set in $X$ such that $U$ be any semi open set containing $A$. Since every semi open set is $\operatorname{sg}$ open, we have $b c l(A) \subset \operatorname{scl}(A) \subset$ $U$. Therefore $\operatorname{bcl}(A) \subset U$. Hence $A$ is $s g^{*} b$-closed set.

The converse of above theorem need not be true as seen from the following example.

Example 3.19. Let $X=\{a, b, c\}$ with $\tau=\{X, \phi,\{b\},\{a, b\}\}$. The set $\{a, b\}$ is $s g^{*} b$ - closed set but not a $s g$-closed set.

\section{Characteristics of $s g^{*} b$-Closed Sets}

Theorem 4.1. If a set $A$ is $s g^{*} b$-closed set then $b c l(A)-A$ contains no non empty sg closed set.

Proof. Let $F$ be a $s g$ closed set in $X$ such that $F \subseteq b c l(A)-A$. Then $A \subseteq X-F$. Since $A$ is $s g^{*} b$-closed set and $X-F$ is $s g$ open then $b c l(A) \subseteq X-F$. (i.e.) $F \subseteq X-b c l(A)$. So $\mathrm{F} \subseteq(X-b c l(A)) \cap(b c l(A)-A)$. Therefore $F=\phi$. 
Theorem 4.2. If $A$ is $s g^{*} b$-closed set in $X$ and $A \subseteq B \subseteq b c l(A)$. Then $B$ is $s g^{*} b$ - closed set in $X$.

Proof. Since $B \subseteq \operatorname{bcl}(A)$, we have $b c l(B) \subseteq \operatorname{bcl}(A)$ then $b c l(B)-B \subseteq$ $b c l(A)-A$. By Theorem 4.1, $b c l(A)-A$ contains no non empty sg closed set. Hence $b c l(B)-B$ contains no non empty $s g$ closed set. Therefore $B$ is $s g^{*} b$-closed set in $X$.

Theorem 4.3. If $A \subseteq Y \subseteq X$ and suppose that $A$ is $s g^{*} b$ closed set in $X$ then $A$ is $s g^{*} b$ - closed set relative to $Y$.

Proof. Given that $A \subseteq Y \subseteq X$ and $A$ is $s g^{*} b$-closed set in $X$. To prove that $A$ is $s g^{*} b$ - closed set relative to $Y$. Let us assume that $A \subseteq Y \cap U$, where $U$ is $s g$ - open in $X$. Since $A$ is $s g^{*} b$ - closed set, $A \subseteq U$ implies $b c l(A) \subseteq U$. It follows that $Y \cap b c l(A) \subseteq Y \cap U$. That is $A$ is $s g^{*} b$ - closed set relative to $Y$.

Theorem 4.4. If $A$ is both $s g$ open and $s g^{*} b$-closed set in $X$, then $A$ is $b$ closed set.

Proof. Since $A$ is $s g$ open and $s g^{*} b$ closed in $X, b c l(A) \subseteq A$. But $A \subseteq$ $b c l(A)$. Therefore $A=b c l(A)$. Hence $A$ is $b$ closed set.

Theorem 4.5. For $x$ in $X$, then the set $X-\{x\}$ is a $s g^{*} b$-closed set or $s g$ open.

Proof. Suppose that $X-\{x\}$ is not $s g$ open, then $X$ is the only $s g$ open set containing $X-\{x\}$. (i.e.) $b c l(X-\{x\}) \subseteq X$. Then $X-\{x\}$ is $s g^{*} b$-closed in $X$.

Note 4.6. $g^{*}$-closed set and $s g^{*} b$-closed set are independent to each other as seen from the following examples

Example 4.7. Let $X=\{a, b, c\}$ with $\tau=\{X, \phi,\{b\},\{c\},\{b, c\}\}$. The set $\{b\}$ is $s g^{*} b$-closed set but not a $g^{*}$-closed set.

Example 4.8. Let $X=\{a, b, c\}$ with $\tau=\{X, \phi,\{c\},\{b, c\}\}$. The set $\{a, c\}$ is $g^{*}$-closed set but not a $s g^{*} b$-closed set.

Note 4.9. $g p$-closed set and $s g^{*} b$ closed set are independent to each other as seen from the following examples.

Example 4.10. Let $X=\{a, b, c\}$ with $\tau=\{X, \phi,\{c\},\{a, c\}\}$. The set $\{b, c\}$ is $g p$-closed set but not a $s g^{*} b$-closed set. 
Example 4.11. Let $X=\{a, b, c\}$ with $\tau=\{X, \phi,\{b\},\{a, b\}\}$. The set $\{a, b\}$ is $s g^{*} b$-closed set but not a gp-closed set.

Note 4.12. $s g^{*} b$ closed set and $g p r$ closed set are independent to each other as seen from the following examples.

Example 4.13. Let $X=\{a, b, c\}$ with $\tau=\{X, \phi,\{a\}\{c\},\{a, c\}\}$. The set $\{a\}$ is $s g^{*} b$-closed set but not a gpr-closed set.

Example 4.14. Let $X=\{a, b, c\}$ with $\tau=\{X, \phi,\{c\},\{a, c\}\}$. The set $\{b, c\}$ is spr-closed set but not a $s g^{*} b$-closed set.

\section{Semi Generalized Star b-Open Sets and Semi Generalized Star $b$-Neighbourhoods}

In this section, we introduce semi generalized star b-open sets (briefly $s g^{*} b$ open) and semi generalized star $b$-neighbourhoods (briefly $s g^{*} b$-neighbourhood) in topological spaces by using the notions of $s g^{*} b$-open sets and study some of their properties.

Definition 5.1. A subset $A$ of a topological space $(X, \tau)$, is called semi generalized star $b$ - open set (briefly $s g^{*} b$-open set) if $A^{c}$ is $s g^{*} b$-closed in $X$. We denote the family of all $s g^{*} b$-open sets in $X$ by $s g^{*} b-O(X)$.

Theorem 5.2. If $A$ and $B$ are $s g^{*} b$-open sets in a space $X$. Then $A \cap B$ is also $s g^{*} b$-open set in $X$.

Proof. If $A$ and $B$ are $s g^{*} b$-open sets in a space $X$. Then $A^{c}$ and $B^{c}$ are $s g^{*} b$-closed sets in a space $X$. By Theorem $4.6 A^{c} \cup B^{c}$ is also $s g^{*} b$-closed set in $X$. (i.e.) $A^{c} \cup B^{c}=(A \cap B)^{c}$ is a $s g^{*} b$-closed set in $X$. Therefore $A \cap B s g^{*} b$-open set in $X$.

Theorem 5.3. If $\operatorname{int}(B) \subseteq B \subseteq A$ and if $A$ is $s g^{*} b$-open in $X$, then $B$ is $s g^{*} b$-open in $X$.

Proof. Suppose that $\operatorname{int}(B) \subseteq B \subseteq A$ and $A$ is $s g^{*} b$-open in $X$ then $A^{c} \subseteq$ $B^{c} \subseteq \operatorname{cl}\left(A^{c}\right)$. Since $A^{c}$ is $s g^{*} b$-closed in $X$, by Theorem $B$ is $s g^{*} b$-open in $X$.

Definition 5.4. Let $x$ be a point in a topological space $X$ and let $x \in X$. A subset $N$ of $X$ is said to be a $s g^{*} b$-neighbourhood of $x$ iff there exists a $s g^{*} b$-open set $G$ such that $x \in G \subset N$. 
Definition 5.5. A subset $N$ of Space $X$ is called a $s g^{*} b$-neighbourhood of $A \subset X$ iff there exists a $s g^{*} b$ - open set $G$ such that $A \subset G \subset N$.

Theorem 5.6. Every neighbourhood $N$ of $x \in X$ is a $s g^{*} b$-neighbourhood of $x$.

Proof. Let $N$ be a neighbourhood of point $x \in X$. To prove that $N$ is a $s g^{*} b$-neighbourhood of $x$. By Definition of neighbourhood, there exists an open set $G$ such that $x \in G \subset N$. Hence $N$ is a $s g^{*} b$-neighbourhood of $x$.

Remark 5.7. In general, a $s g^{*} b$-neighbourhood of $x \in X$ need not be a neighbourhood of $x$ in $X$ as seen from the following example.

Example 5.8. Let $X=\{a, b, c\}$ with topology $\tau=\{X, \phi,\{c\}$, $\{a, c\}\}$. Then $s g^{*} b-O(X)=\{X, \phi,\{c\},\{a, c\},\{b, c\}\}$. The set $\{b, c\}$ is $s g^{*} b$ neighbourhood of point $c$, since the $s g^{*} b$-open sets $\{c\}$ is such that $c \in\{c\} \subset$ $\{b, c\}$. However, the set $\{b, c\}$ is not a neighbourhood of the point $c$, since no open set $G$ exists such that $c \in G \subset\{b, c\}$.

Remark 5.9. The $s g^{*} b$-neighbourhood $N$ of $x \in X$ need not be a $s g^{*} b$ open in $X$.

Theorem 5.10. If a subset $N$ of a space $X$ is $s g^{*} b$-open, then $N$ is $s g^{*} b$-neighbourhood of each of its points.

Proof. Suppose $N$ is $s g^{*} b$-open. Let $x \in N$. We claim that $N$ is $s g^{*} b_{\text {- }}$ neighbourhood of $x$. For $N$ is a $s g^{*} b$-open set such that $x \in N \subset N$. Since $x$ is an arbitrary point of $N$, it follows that $N$ is a $s g^{*} b$-neighbourhood of each of its points.

Theorem 5.11. Let $X$ be a topological space. If $F$ is $s g^{*} b$-closed subset of $X$ and $x \in F^{c}$. Prove that there exists a $s g^{*} b$-neighbourhood $N$ of $x$ such that $N \cap F=\phi$.

Proof. Let $F$ be $s g^{*} b$-closed subset of $X$ and $x \in F^{c}$. Then $F^{c}$ is $s g^{*} b$-open set of $X$. So by Theorem $5.10 F^{c}$ contains a $s g^{*} b$-neighbourhood of each of its points. Hence there exists a $s g^{*} b$-neighbourhood $N$ of $x$ such that $N \subset F^{c}$. (i.e.) $N \cap F=\phi$.

Definition 5.12. Let $x$ be a point in a topological space $X$. The set of all $s g^{*} b$-neighbourhood of $x$ is called the $s g^{*} b$-neighbourhood system at $x$, and is denoted by $s g^{*} b-N(x)$. 
Theorem 5.13. Let a $s g^{*} b$-neighbourhood $N$ of $X$ be a topological space and each $x \in X$, Let $s g^{*} b-N(X, \tau)$ be the collection of all $s g^{*} b$-neighbourhood of $x$. Then we have the following results.

(i) $x \in, s g^{*} b-N(x) \neq \phi$.

(ii) $N \in s g^{*} b-N(x) \Rightarrow x \in N$.

(iii) $N \in s g^{*} b-N(x), M \supset N \Rightarrow M \in s g^{*} b-N(x)$.

(iv) $N \in s g^{*} b-N(x), M \in s g^{*} b-N(x) \Rightarrow N \cap M \in s g^{*} b-N(x)$, if finite intersection of $s g^{*} b$ open set is $s g^{*} b$ open.

(v) $N \in s g^{*} b-N(x) \Rightarrow$ there exists $M \in s g^{*} b-N(x)$ such that $M \subset N$ and $M \in s g^{*} b-N(y)$ for every $y \in M$.

Proof. 1. Since $X$ is $s g^{*} b$-open set, it is a $s g^{*} b$-neighbourhood of every $x \in X$. Hence there exists at least one $s g^{*} b$-neighbourhood (namely- $X$ ) for each $x \in X$. Therefore $s g^{*} b-N(x) \neq \phi$ for every $x \in X$.

2. If $N \in s g^{*} b-N(x)$, then $N$ is $s g^{*} b$-neighbourhood of $x$. By Definition of $s g^{*} b$-neighbourhood, $x \in N$.

3. Let $N \in s g^{*} b-N(x)$ and $M \supset N$. Then there is a $s g^{*} b$-open set $G$ such that $x \in G \subset N$. Since $N \subset M, x \in G \subset M$ and so $M$ is $s g^{*} b_{-}$ neighbourhood of $x$. Hence $M \in s g^{*} b-N(x)$.

4. Let $N \in s g^{*} b-N(x), M \in s g^{*} b-N(x)$. Then by Definition of $s g^{*} b$ neighbourhood, there exists $s g^{*} b$ - open sets $G_{1}$ and $G_{2}$ such that $x \in$ $G_{1} \subset N$ and $x \in G_{2} \subset M$. Hence

$$
x \in G_{1} \cap G_{2} \subset N \cap M
$$

Since $G_{1} \cap G_{2}$ is a $s g^{*} b$-open set, it follows from (1) that $N \cap M$ is a $s g^{*} b$ neighbourhood of $x$.

Hence $N \cap M \in s g^{*} b-N(x)$.

5. Let $N \in s g^{*} b-N(x)$, Then there is a $s g^{*} b$-open set $M$ such that $x \in$ $M \subset N$. Since $M$ is $s g^{*} b$-open set, it is $s g^{*} b$-neighbourhood of each of its points.

Therefore $M \in s g^{*} b-N(y)$ for every $y \in M$. 


\section{References}

[1] M.E. Abd El-Monsef, S.N. Deeb and R.A. Mahmoud, $\beta$-open sets and $\beta$ continous mappings, Bull.Fac.Sci.Assiut, Univ, 12(1983), 77-90.

[2] Ahmad Al-Omari and Mohd. Salmi Md. Noorani, On Generalized b-closed sets, Bull. Malays. Math. Sci. Soc(2) 32(1) (2009), 19-30

[3] D.Andrijevic, Semi-pre open sets, Mat.Vesnik 38 (1) 1986, 24-32.

[4] D.Andrijevic, b-open sets, Mat.Vesink, 48 (1996), 59-64.

[5] S.P.Arya and T.Nour, Characterizations of s-normal spaces, Indian J.Pure Applied Maths 21 (8) (1990) 717-719.

[6] P.Bhattacharya and B.K.Lahiri, Semi-generalized closed sets on topology, Indian J.Maths 29 (3) (1987) 375-382.

[7] M.Caldas and S.Jafari, On some applications of b-open sets in topological spaces, Kochi J.Math. 24(4) (1998), 681-688.

[8] G.Dimaio and T.Noiri, On s-closed spaces, Indian J-pure appl.math 18 (3) 1987, 226-233

[9] J.Dontchev, On generalized semi- pre open sets, Mem. Fac. Sci. Kochi. Univ. ser. A. math 16 (1995) 35.

[10] Y.Gnanambal, On generalized pre-regular closed sets in topological spaces, Indian J.Pure Appl, Math 28 (1997), 351-360

[11] D. Iyappan \& N.Nagaveni, On semi generalized b-closed set, Nat. Sem. On Mat \& Comp.Sci, Jan (2010), Proc.6

[12] N.Levine, Generalized closed sets in topology, Tend Circ., Mat. Palermo (2) 19 (1970), 89-96.

[13] N.Levine, Semi-open sets and semi-continuity in topological spaces, Amer. Math. Monthly 70 (1963)), 36-41

[14] H,.Maki, R.Devi and K.Balachandran, Associated topologies of generalized $\alpha$-closed sets and $\alpha$-generalized closed sets, Mem. Fac. Sci. Kochi. Univ. Ser. A.Math. 15 (1994), 5163.

[15] H.Maki, R.J.Umehara and T.Noiri, Every topological space is pre-T 1/2, Mem. Fac. Sci. Kochi. Univ. Ser. A. Math. 17(1996), 33-42

[16] A.S.Mashor Abd.El-Monsef.M.E and Ei-Deeb.S.N., On Pre continuous and weak precontinuous mapping, Proc.Math.,Phys.Soc.Egypt, 53 (1982), 47-53.

[17] O.Njastad, On some classes of nearly open sets, Pacific J Math., 15(1965), 961-970.

[18] N.Nagaveni, Studies on generalized on homeomorphisms in topological spaces, Ph.D Thesis, Bharathiar University, Coimbatore 1999

[19] Ravi.O and Ganesan.S, g-closed sets in Topology, International Journal of Computer Science \& Emerging Technologies, Vol 2, Issue3, June 2011, 330-337.

[20] Veerakumar.M.K.R.S., Between closed sets and g-closed sets, Mem.Fac.Sci.Kochi.Univ.Ser.A, Math, 21 (2000), 1-19. 